\title{
Inequalities between Lattice Packing and Covering Densities of Centrally Symmetric Plane Convex Bodies
}

\author{
D. Ismailescu \\ Courant Institute of Mathematical Sciences, New York University, \\ 251 Mercer Street, New York, NY 10012, USA \\ ismailes@cims.nyu.edu
}

\begin{abstract}
Given a family $\mathcal{C}$ of plane convex bodies, let $\Omega(\mathcal{C})$ be the set of all pairs $(x, y)$ with the property that there exists $K \in \mathcal{C}$ such that $\vartheta(K)=x$ and $\delta(K)=y$, where $\vartheta(K)$ and $\delta(K)$ denote the densities of the thinnest covering and the densest packing of the plane with copies of $K$, respectively. The set $\Omega_{\mathrm{L}}(\mathcal{C})$ is defined analogously, with the difference that we restrict our attention to lattice packings and coverings.

We prove that, for every centrally symmetric plane convex body $K$,

$$
1-\delta_{\mathrm{L}}(K) \leq \vartheta_{\mathrm{L}}(K)-1 \leq 1.25 \sqrt{1-\delta_{\mathrm{L}}(K)}
$$

and give an exact analytic description of $\Omega_{\mathrm{L}}\left(\mathcal{P}_{8}\right)$ where $\mathcal{P}_{8}$ is the family of all centrally symmetric octagons. This allows us to show that the above inequalities are asymptotically tight.
\end{abstract}

\section{Introduction}

Throughout this paper we consider arrangements of convex bodies in the Euclidean plane. A convex body is a compact convex set with nonempty interior; its area is denoted by $A(K)$. An arrangement of congruent replicas (translates) of a convex body $K$ is a family $\mathcal{A}$ of convex bodies, each of which is congruent to (is a translate of) $K$. The arrangement is a packing if its members' interiors are mutually disjoint, and it is a covering if the union of its members is the whole plane. For any pair of independent vectors $\mathbf{u}$ and $\mathbf{v}$ in $E$, the lattice generated by $\mathbf{u}$ and $\mathbf{v}$ is the set of vectors $L(\mathbf{u}, \mathbf{v})=\{n \mathbf{u}+m \mathbf{v}: n, m$ integers $\}$. An arrangement of translates of $K$ by all vectors of a lattice is a lattice arrangement. Thus we may have lattice packings and lattice coverings of $E$ with translates of $K$.

The density of an arrangement $\mathcal{A}$ is, intuitively speaking, the ratio between the sum of the areas of members of $\mathcal{A}$ contained in an arbitrarily large region and the area of the region and is rigorously defined by an appropriate limit (see [3], [8], and [13]). 
Given a convex body $K$, the packing density of $K$, denoted by $\delta(K)$, is the maximum density of any packing with congruent replicas of $K$. If we restrict ourselves to packings with translates of $K$, or just to lattice packings, then the maximum density defines the translational packing density of $K$ or the lattice packing density of $K$, denoted by $\delta_{\mathrm{T}}(K)$ and by $\delta_{\mathrm{L}}(K)$, respectively. The analogous notions of the covering density, translational covering density, and lattice covering density denoted by $\vartheta(K), \vartheta_{\mathrm{T}}(K)$, and $\vartheta_{\mathrm{L}}(K)$, respectively, are defined as the minimum density of coverings of a corresponding type.

Obviously, the following inequalities hold for every convex body $K$ :

$$
\delta_{\mathrm{L}}(K) \leq \delta_{\mathrm{T}}(K) \leq \delta(K) \leq 1 \leq \vartheta(K) \leq \vartheta_{\mathrm{T}}(K) \leq \vartheta_{\mathrm{L}}(K)
$$

For a given convex body $K$, let $H_{\min }$ denote a hexagon of minimum area which contains $K$ and let $h_{\max }$ denote a hexagon of maximum area which is contained in $K$.

L. Fejes-Tóth [5] proved that for every convex body $K$ we have

$$
\delta(K) \leq A(K) / A\left(H_{\min }\right) \quad \text { and } \quad \vartheta_{\mathrm{T}}(K) \geq A(K) / A\left(h_{\max }\right)
$$

By a theorem of Dowker [1], if $K$ is centrally symmetric, then $h_{\max }$ and $H_{\min }$ can be obtained by inscribing in, resp. circumscribing about, $K$ a centrally symmetric hexagon. Since translates of any centrally symmetric hexagon can be arranged to form a plane lattice tiling, it immediately follows that, for each centrally symmetric convex body $K$, we have

$$
\begin{aligned}
\delta(K) & =\delta_{\mathrm{L}}(K)=A(K) / A\left(H_{\min }\right), \\
\vartheta_{\mathrm{T}}(K) & =\vartheta_{\mathrm{L}}(K)=A(K) / A\left(h_{\max }\right) .
\end{aligned}
$$

It is conjectured (see p. 205 of [6]) that $\vartheta(K)=\vartheta_{\mathrm{L}}(K)$ holds for all centrally symmetric plane convex bodies $K$; this conjecture is supported by the partial result of [5] about crossing-free coverings.

Improving previous results of Mahler and Ennola [12], [2], Tammela [17] proved that under the assumption of central symmetry for $K$ we have

$$
\delta_{\mathrm{L}}(K) \geq 0.892656 \cdots
$$

The conjectured greatest lower bound for $\delta_{\mathrm{L}}(K)$ over all centrally symmetric convex bodies $K$ is

$$
\delta_{\mathrm{L}}\left(K_{0}\right)=(8-\sqrt{32}-\ln 2) /(\sqrt{8}-1)=0.90241418 \ldots
$$

attained by the so-called "smoothed-octagon" $K_{0}$ constructed by Reinhardt in [14].

The analogous problem for coverings in the case of central symmetry of $K$ has been settled as a corollary of a result proved by Sas [15]. More precisely, the inequality

$$
\vartheta_{\mathrm{L}}(K) \leq 2 \pi / \sqrt{27}
$$


holds for each centrally symmetric convex body $K$, and the equality occurs if and only if $K$ is an ellipse, which shows that the above inequality is sharp.

We mention another result of L. Fejes-Tóth (see [7]) which states that, for every centrally symmetric convex body $K$,

$$
\delta_{\mathrm{L}}(K) / \vartheta_{\mathrm{L}}(K) \geq \frac{3}{4} .
$$

The following theorem is due to Schmidt ([16], see also [3]) and it is used implicitly several times throughout the paper.

Theorem (Schmidt). Given a convex body $K$, the following conditions are equivalent:

(i) $\delta(K)=1$;

(ii) $\vartheta(K)=1$;

(iii) $K$ is a tile.

The reader interested in a more comprehensive account of the theory of packing and covering, is referred to the survey papers of G. Fejes-Tóth and Kuperberg [3], [4] and to the book by Pach and Agarwal [13].

\section{The Problem and Summary of Results}

Given a family $\mathcal{C}$ of plane convex bodies let $\Omega(\mathcal{C})$ be the set of all pairs $(x, y)$ with the property that there exists $K \in \mathcal{C}$ such that $\vartheta(K)=x$ and $\delta(K)=y$; the set $\Omega_{\mathrm{L}}(\mathcal{C})$ is defined analogously, with the difference that we restrict our attention to lattice packings and coverings.

The problem of determining $\Omega(\mathcal{C})$ and $\Omega_{\mathrm{L}}(\mathcal{C})$ is undoubtedly a very difficult one, even if we restrict $\mathcal{C}$ to be the family of all centrally symmetric convex bodies (see for instance the gap between the estimates (3) and (4)).

Questions regarding the sets $\Omega(\mathcal{C})$ and $\Omega_{\mathrm{L}}(\mathcal{C})$, where $\mathcal{C}$ is the set of all convex bodies, were raised by $\mathrm{G}$. Fejes-Tóth and Kuperberg in [4] and [10].

Since every centrally symmetric convex body can be approximated arbitrarily close by a centrally symmetric polygon, a natural approach is trying to determine $\Omega_{\mathrm{L}}\left(\mathcal{P}_{2 n}\right)$ where $\mathcal{P}_{2 n}$ is the class of all centrally symmetric convex polygons with $2 n$ vertices.

Obviously, $\Omega_{\mathrm{L}}\left(\mathcal{P}_{4}\right)=\Omega_{\mathrm{L}}\left(\mathcal{P}_{6}\right)=\{(1,1)\}$ since every parallelogram or centrally symmetric hexagon tiles the plane in a lattice manner.

Our first result gives $\Omega_{L}\left(\mathcal{P}_{8}\right)$.

\section{Theorem 1.}

$$
\Omega_{\mathrm{L}}\left(\mathcal{P}_{8}\right)=U
$$

where $U$ is the set

$$
\left\{(\vartheta, \delta): 1 \leq \vartheta \leq 4-2 \sqrt{2}, \frac{5 \vartheta^{2}-12 \vartheta+8}{2 \vartheta^{2}-5 \vartheta+4} \leq \delta \leq \frac{\vartheta\left(\vartheta+4+\sqrt{\vartheta^{2}-8 \vartheta+8}\right)}{4 \vartheta+2}\right\}
$$


The shape of $\Omega_{\mathrm{L}}\left(\mathcal{P}_{8}\right)$ (see the last section) suggested the next two theorems:

Theorem 2. For every centrally symmetric plane convex body $K$,

$$
\vartheta_{\mathrm{L}}(K)-1 \geq 1-\delta_{\mathrm{L}}(K) \text {. }
$$

Theorem 3. For every centrally symmetric plane convex body $K$,

$$
\vartheta_{\mathrm{L}}(K)-1 \leq 1.25 \sqrt{1-\delta_{\mathrm{L}}(K)}
$$

Finally, the following theorems show that the orders of magnitude in the above inequalities cannot be improved.

Theorem 4. For every $\varepsilon>0$ there exists a centrally symmetric plane convex body $K_{\varepsilon}$ such that

$$
\vartheta_{\mathrm{L}}\left(K_{\varepsilon}\right)-1<(1+\varepsilon)\left(1-\delta_{\mathrm{L}}\left(K_{\varepsilon}\right)\right)
$$

Theorem 5. For every $\varepsilon>0$ there exists a centrally symmetric plane convex body $K_{\varepsilon}$ such that

$$
\vartheta_{\mathrm{L}}\left(K_{\varepsilon}\right)-1>(1-\varepsilon) \sqrt{1-\delta_{\mathrm{L}}\left(K_{\varepsilon}\right)}
$$

The proofs are presented in the subsequent sections.

\section{3. $\delta_{\mathrm{L}}(K)$ and $\vartheta_{\mathrm{L}}(K)$ in the Case of an Octagon}

Let $K=O_{\mathrm{c}} \in \mathcal{P}_{8}$ be an octagon having the origin as center of symmetry. Following an idea of Mahler (see [11]), it is convenient for our purposes to specify the octagon by the vectors

$$
\mathbf{r}_{1}, \mathbf{r}_{2}, \mathbf{r}_{3}, \mathbf{r}_{4},-\mathbf{r}_{1},-\mathbf{r}_{2},-\mathbf{r}_{3},-\mathbf{r}_{4}
$$

which form the sides of the polygon; thus $\mathbf{r}_{i}=\overline{P_{i} P_{i+1}}$.

The outer product

$$
a_{i j}=\left(\mathbf{r}_{i}, \mathbf{r}_{j}\right)
$$

represents the signed area of the parallelogram made by vectors $\mathbf{r}_{i}$ and $\mathbf{r}_{j}$.

We recall the basic properties of the outer product:

(a) Skew-symmetry: $(\mathbf{u}, \mathbf{v})=-(\mathbf{v}, \mathbf{u})$.

(b) Linearity: $(a \mathbf{u}, \mathbf{v})=a \cdot(\mathbf{u}, \mathbf{v})$ and $(\mathbf{u}, \mathbf{v}+\mathbf{w})=(\mathbf{u}, \mathbf{v})+(\mathbf{u}, \mathbf{w})$.

(c) Nondegeneracy: $(\mathbf{u}, \mathbf{v})=0$ if and only if $\mathbf{u}$ and $\mathbf{v}$ are dependent.

For an elementary presentation on the geometrical applications of the outer product the reader may consult [9]. 


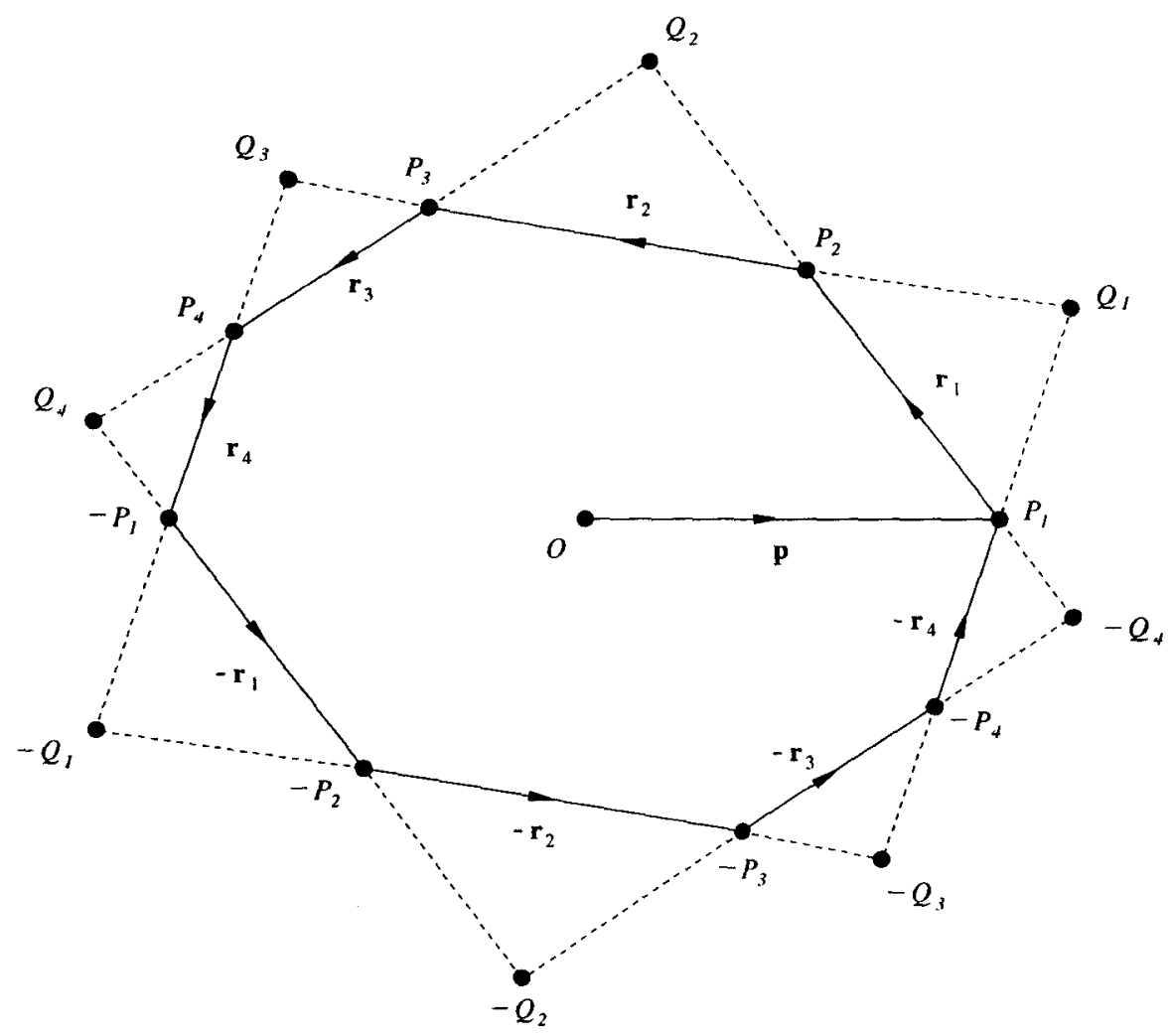

Fig. 1. A centrally symmetric convex octagon.

The octagon is convex if and only if (see Fig. 1)

$$
a_{i j}>0 \quad(1 \leq i<j \leq 4) .
$$

It is important to note that the quantities $a_{i j}$ are not independent. Namely,

$$
a_{12} a_{34}+a_{14} a_{23}=a_{13} a_{24} \text {. }
$$

For, since any three vectors in the plane are linearly dependent,

$$
\mathbf{r}_{3}=\lambda \mathbf{r}_{1}+\mu \mathbf{r}_{2} \quad(\lambda, \mu \text { scalars })
$$

On forming the outer product with $\mathbf{r}_{1}$ and $\mathbf{r}_{2}$, it is found that

$$
a_{13}=\mu a_{12}, \quad a_{23}=-\lambda a_{12}
$$

and therefore

$$
a_{23} \mathbf{r}_{1}-a_{13} \mathbf{r}_{2}+a_{12} \mathbf{r}_{3}=0,
$$

whence, on multiplying by $\mathbf{r}_{4}$, we obtain (13). 
Thus there are five independent coefficients $a_{i j}$ which determine the octagon apart from affine transformations.

By Fig. 1 the position vector of the vertex $P_{i}$ is $\mathbf{p}+\mathbf{r}_{1}+\cdots+\mathbf{r}_{i-1}(i=1,2,3,4$ : $\left.\mathbf{r}_{0}=\mathbf{0}\right)$ where $\mathbf{p}=-\frac{1}{2}\left(\mathbf{r}_{1}+\mathbf{r}_{2}+\mathbf{r}_{3}+\mathbf{r}_{4}\right)$.

Hence,

$$
A\left(O P_{i} P_{i+1}\right)=\frac{1}{2}\left(\mathbf{p}+\mathbf{r}_{1}+\cdots+\mathbf{r}_{i-1}, \mathbf{r}_{i}\right)=\frac{1}{2}\left(\mathbf{p}, \mathbf{r}_{i}\right)+\frac{1}{2} \sum_{k=1}^{i-1}\left(\mathbf{r}_{k}, \mathbf{r}_{i}\right)
$$

and

$$
A\left(O_{\mathrm{c}}\right)=2 \sum A\left(O P_{i} P_{i+1}\right)=\left(\mathbf{p}, \sum_{i=1}^{4} \mathbf{r}_{i}\right)+\sum_{1 \leq i<j \leq 4}\left(\mathbf{r}_{i}, \mathbf{r}_{j}\right)
$$

that is,

$$
A\left(O_{\mathrm{c}}\right)=\sum_{1 \leq i<j \leq 4} a_{i j}=a_{12}+a_{13}+a_{14}+a_{23}+a_{24}+a_{34}
$$

The subsequent argument is concerned with the symmetrical hexagons that can be circumscribed to $O_{\mathrm{c}}$. Since we are interested in using $H_{\min }$, a hexagon of minimum area circumscribed about $O_{\mathrm{c}}$, a simple argument shows that we can restrict ourselves to considering only those hexagons which are obtained by leaving out one pair of parallel sides from the original octagon. Denote the hexagon obtained by omitting $P_{i} P_{i+1}$ and $\left(-P_{i}\right)\left(-P_{i+1}\right)$ by $H_{i}$.

The area of $H_{i}$ (see Fig. 1) exceeds the area of $O_{\mathrm{c}}$ by

$$
2 A\left(P_{i} Q_{i} P_{i+1}\right)=\frac{a_{i-1, i} a_{i, i+1}}{a_{i-1, i+1}} .
$$

For $2 A\left(P_{i} Q_{i} P_{i+1}\right)=\left(\overline{P_{i} Q_{i}}, \mathbf{r}_{i}\right)=\lambda_{i}\left(\mathbf{r}_{i-1}, \mathbf{r}_{i}\right)=\lambda_{i} a_{i-1, i}$ where $\lambda_{i}$ is a scalar which is determined by the condition that $\lambda_{i} \mathbf{r}_{i-1}-\mathbf{r}_{i}$ should be parallel to $\mathbf{r}_{i+1}$. Therefore,

$$
0=\left(\lambda_{i} \mathbf{r}_{i-1}-\mathbf{r}_{i}, \mathbf{r}_{i+1}\right)=\lambda_{i} a_{i-1, i+1}-a_{i, i+1}
$$

from which (15) follows.

Now using (1) it follows that

$$
\delta_{\mathrm{L}}\left(O_{\mathrm{c}}\right)=\frac{A\left(O_{\mathrm{c}}\right)}{A\left(O_{\mathrm{c}}\right)+m}, \quad \text { where } \quad m=\min \left\{\frac{a_{12} a_{14}}{a_{24}}, \frac{a_{12} a_{23}}{a_{13}}, \frac{a_{23} a_{34}}{a_{24}}, \frac{a_{34} a_{14}}{a_{13}}\right\} .
$$

A similar discussion could be had for $h_{\max }$, the symmetrical hexagon(s) of maximum area which can be inscribed in $O_{\mathrm{c}}$. A simple analysis shows that it is sufficient to consider only four hexagons, each being obtained by leaving out one pair of opposite vertices, say $P_{i}$ and $-P_{i}$, of the initial octagon. Denote this hexagon by $h_{i}$.

The area of $O_{\mathrm{c}}$ exceeds the area of $h_{i}$ by $2 A\left(P_{i-1} P_{i} P_{i+1}\right)=a_{i-1, i}$.

From (2) it follows that

$$
\vartheta_{\mathrm{L}}\left(O_{\mathrm{c}}\right)=\frac{A\left(O_{c}\right)}{A\left(O_{\mathrm{c}}\right)-M}, \quad \text { where } \quad M=\min \left\{a_{12}, a_{23}, a_{34}, a_{14}\right\}
$$




\section{Proof of Theorem 1}

The proof is presented in the following two subsections.

\section{1. $U \subseteq \Omega_{\mathrm{L}}\left(\mathcal{P}_{8}\right)$}

Given any $(\vartheta, \delta) \in U$ we will construct a (possibly degenerate) centrally symmetric octagon $O_{\mathrm{c}}$ for which $\vartheta_{\mathrm{L}}\left(O_{\mathrm{c}}\right)=\vartheta$ and $\delta_{\mathrm{L}}\left(O_{\mathrm{c}}\right)=\delta$. If $(\vartheta, \delta)=(1,1)$, then every centrally symmetric hexagon will do. In what follows we consider only the points $(\vartheta, \delta) \in$ $U$ for which $\delta<1<\vartheta$.

As noticed before, a convex centrally symmetric octagon is uniquely determined up to an affine transformation by the associated positive quantities $a_{i j}, 1 \leq i<j \leq 4$, subject to the relation (13).

Define the octagon $O_{\mathrm{c}}$ by setting

$$
\begin{aligned}
& a_{12}=a_{34}=1 \text {, } \\
& a_{23}=a_{14}=\alpha \text {, } \\
& a_{24}=\frac{\delta(\vartheta-1) \alpha}{\vartheta(1-\delta)}, \quad a_{13}=\frac{\vartheta(1-\delta)\left(\alpha^{2}+1\right)}{\delta(\vartheta-1) \alpha},
\end{aligned}
$$

where $\alpha$ is the greatest root of the quadratic equation

$$
x^{2} \cdot(\vartheta-\delta)^{2}-x \cdot \delta \vartheta(1-\delta)(2-\vartheta)+\vartheta^{2}(1-\delta)^{2}=0
$$

that is,

$$
\alpha=\frac{(1-\delta) \vartheta[\delta(2-\vartheta)+\sqrt{\vartheta(2-\delta)(4 \delta-\delta \vartheta-2 \vartheta)}]}{2(\vartheta-\delta)^{2}}
$$

It is an easy matter to check that since $(\vartheta, \delta) \in U$ the quadratic equation from (19) has real roots.

Claim A. For every $(\vartheta, \delta) \in U$ and using the above notations we have that

$$
\begin{aligned}
\min \left\{a_{12}, a_{23}, a_{34}, a_{14}\right\} & =1 \\
\min \left\{\frac{a_{12} a_{14}}{a_{24}}, \frac{a_{12} a_{23}}{a_{13}}, \frac{a_{23} a_{34}}{a_{24}}, \frac{a_{34} a_{14}}{a_{13}}\right\} & =\frac{\vartheta(1-\delta)}{\delta(\vartheta-1)} .
\end{aligned}
$$

The proof of this claim is given in the Appendix.

It is now possible, using (14), (16)-(18), and the above claim, to express $\delta_{\mathrm{L}}\left(O_{\mathrm{c}}\right)$ and $\vartheta_{\mathrm{L}}\left(O_{\mathrm{c}}\right)$ in terms of $\delta, \vartheta$, and $\alpha$. It is a simple exercise to show that both equalities $\delta_{\mathrm{L}}\left(O_{\mathrm{c}}\right)=\delta$ and $\vartheta_{\mathrm{L}}\left(O_{\mathrm{c}}\right)=\vartheta$ are equivalent to the following relation:

$$
\alpha^{2}(\vartheta-\delta)^{2}-\alpha \delta \vartheta(1-\delta)(2-\vartheta)+\vartheta^{2}(1-\delta)^{2}=0
$$

However, this is true from our choice of $\alpha$ (see (19)).

It follows that $U \subseteq \Omega_{\mathrm{L}}\left(\mathcal{P}_{8}\right)$. 


\section{2. $\Omega_{\mathrm{L}}\left(\mathcal{P}_{8}\right) \subseteq U$}

We have to show that given any centrally symmetric octagon $O$ it is true that $\left(\vartheta_{\mathrm{L}}(O)\right.$, $\delta_{\mathrm{L}}(O)$ ) belongs to $U$. The problem is trivial if $O$ is degenerate, since in this case $\left(\vartheta_{\mathrm{L}}(O), \delta_{\mathrm{L}}(O)\right)=(1,1) \in U$.

Similarly as above, consider the positive quantities $a_{i j}, 1 \leq i<j \leq 4$, satisfying (13), which determine $O$ up to an affine transformation. Without losing any generality we can assume that

$$
\min \left\{a_{12}, a_{23}, a_{34}, a_{14}\right\}=a_{12} .
$$

For the remainder of this section we use the following notations for the $a_{i j}$ 's corresponding to our octagon $O$ :

$$
\begin{aligned}
& a_{12}=x, \quad a_{23}=x+a, \quad a_{34}=x+b, \quad a_{14}=x+c, \quad a_{13}=y, \quad a_{24}=z, \\
& \text { where } \quad x>0, \quad a \geq 0, \quad b \geq 0, \quad c \geq 0, \quad \text { and } y z=2 x^{2}+x(a+b+c)+a c,
\end{aligned}
$$

the inequalities coming from our assumption (23) and the equality is relation (13) written in the new variables.

Also, we introduce two more notations:

$$
\begin{aligned}
t & =y+z+a+b+c \\
m & =\min \left\{\frac{x(x+a)}{y}, \frac{(x+a)(x+b)}{z}, \frac{(x+b)(x+c)}{y}, \frac{(x+c) x}{z}\right\} .
\end{aligned}
$$

Finally, in order to improve the readability, during this section $\delta$ and $\vartheta$ denote $\delta_{\mathrm{L}}(O)$ and $\vartheta_{\mathrm{L}}(O)$, respectively. With these new variables, using (14), we obtain the following equivalent forms of (16) and (17):

$$
\delta=\frac{4 x+t}{4 x+t+m} \quad \text { and } \quad \vartheta=\frac{4 x+t}{3 x+t} .
$$

We divide the proof of $\Omega_{\mathrm{L}}\left(\mathcal{P}_{8}\right) \subseteq U$ into three steps.

Step 1 .

$1 \leq \vartheta \leq 4-2 \sqrt{2}$. The left inequality is trivial. For the right one notice that (27) gives

$$
\frac{1}{\vartheta-1}=3+\frac{t}{x}=3+\frac{y+z+a+b+c}{x} \geq 3+\frac{2 \sqrt{y z}+a+b+c}{x},
$$

which taking into account (24) implies that

$$
\frac{1}{\vartheta-1} \geq 3+\frac{2 \sqrt{2 x^{2}+x(a+b+c)+a c}+a+b+c}{x} \geq 3+\frac{2 \sqrt{2 x^{2}}}{x},
$$

thus, $1 /(\vartheta-1) \geq 3+2 \sqrt{2}$, which immediately implies the desired inequality.

Step 2.

$$
\delta \geq \frac{5 \vartheta^{2}-12 \vartheta+8}{2 \vartheta^{2}-5 \vartheta+4}
$$


Equation (27) gives that

$$
m=\frac{1-\delta}{\delta} \cdot(4 x+t)
$$

Since the function $f(\delta)=(1-\delta) / \delta$ is decreasing, (28) is equivalent to

$$
f(\delta) \leq f\left(\frac{5 \vartheta^{2}-12 \vartheta+8}{2 \vartheta^{2}-5 \vartheta+4}\right) \Leftrightarrow \frac{1-\delta}{\delta} \leq \frac{-3 \vartheta^{2}+7 \vartheta-4}{5 \vartheta^{2}-12 \vartheta+8},
$$

which after using (27) and (29) can be written as

$$
m \leq \frac{(4 x+t) x t}{8 x^{2}+4 x t+t^{2}}
$$

Suppose that (30) is not always true. Using (26) it then follows that there exist values of $x, y, z, a, b, c$ satisfying the restrictions in (24) such that

$$
\begin{aligned}
& \frac{x(x+a)}{y}>\frac{(4 x+t) x t}{8 x^{2}+4 x t+t^{2}} \\
& \frac{x(x+c)}{z}>\frac{(4 x+t) x t}{8 x^{2}+4 x t+t^{2}} .
\end{aligned}
$$

Multiplying the last two inequalities it follows that

$$
\frac{(x+a)(x+c)}{y z}>\left[\frac{(4 x+t) t}{8 x^{2}+4 x t+t^{2}}\right]^{2} .
$$

We will show that (31) leads to a contradiction. This would end Step 2.

Using the fact that $y z=(x+a)(x+c)+x(x+b),(31)$ gives

$$
\begin{gathered}
\frac{(x+a)(x+c)}{(x+a)(x+c)+x(x+b)}>\left[\frac{(4 x+t) t}{(4 x+t) t+8 x^{2}}\right]^{2} \\
\Rightarrow 1+\frac{x(x+b)}{(x+a)(x+c)}<\left[1+\frac{8 x^{2}}{(4 x+t) t}\right]^{2}
\end{gathered}
$$

and after some computations

$$
\frac{16 x(x+a)(x+c)}{x+b}>\left[\frac{(4 x+t) t}{2 x+t}\right]^{2}
$$

Suppose now that $x, a, b, c$ are fixed and $y, z$ are variable subject to the restrictions $y>0, z>0$, and $y z=2 x^{2}+x(a+b+c)+a c$.

It is an easy exercise to see that the function $g(t)=(4 x+t) t /(2 x+t)$ is increasing in $t$ and hence

$$
g(a+b+c+y+z) \geq g(a+b+c+2 \sqrt{y z}) .
$$

Now, (32) and the above argument imply that

$$
\frac{16 x(x+a)(x+c)}{x+b}>\left[\frac{\left(4 x+t_{0}\right) t_{0}}{2 x+t_{0}}\right]^{2}
$$

where $t_{0}=a+b+c+2 \sqrt{2 x^{2}+x(a+b+c)+a c}$. 
We now consider $x, a, c$ being fixed and $b \geq 0$ as a variable. An immediate calculation shows that the right term of (33) increases with $b$ while obviously the left term decreases. Therefore, setting $b=0$ in (33) will imply

$$
16(x+a)(x+c)>\left[\frac{\left(4 x+t_{1}\right) t_{1}}{2 x+t_{1}}\right]^{2},
$$

where $t_{1}=a+c+2 \sqrt{2 x^{2}+x(a+c)+a c}$.

We introduce the following notations:

$$
u=2 x+a+c \text { and } v=\sqrt{2 x^{2}+x(a+c)+a c} .
$$

Straightforward computations immediately give that

$$
\begin{aligned}
4(x+a)(x+c) & =u^{2}-(a-c)^{2} \\
2 x+t_{1} & =u+2 v \\
\left(4 x+t_{1}\right) t_{1} & =2 u^{2}+4 u v-(a-c)^{2} .
\end{aligned}
$$

From (34) and the above equalities it follows that

$$
4\left[u^{2}-(a-c)^{2}\right] \cdot(u+2 v)^{2}>\left[2 u^{2}+4 u v-(a-c)^{2}\right],
$$

which after expanding and simplifying gives

$$
-(a-c)^{2} \cdot\left[16 v^{2}+8 u v+(a-c)^{2}\right]>0 .
$$

This last relation is clearly impossible. This ends Step 2.

Step 3.

$$
\delta \leq \frac{\vartheta\left(\vartheta+4+\sqrt{\vartheta^{2}-8 \vartheta+8}\right)}{4 \vartheta+2} .
$$

Suppose that the opposite inequality could be true. Using the expressions of $\delta$ and $\vartheta$ given by (27) it follows after some calculations that it is possible to have

$$
m<\frac{t-\sqrt{t^{2}-8 x^{2}}}{4}
$$

Now using the definition of $m$ from (26), the previous inequality implies that

$$
\min \left\{\frac{x(x+a)}{y}, \frac{(x+a)(x+b)}{z}, \frac{(x+b)(x+c)}{y}, \frac{(x+c) x}{z}\right\}<\frac{2 x^{2}}{t+\sqrt{t^{2}-8 x^{2}}},
$$

which obviously gives

$$
\min \left\{\frac{x^{2}}{y}, \frac{x^{2}}{z}\right\}<\frac{2 x^{2}}{t+\sqrt{t^{2}-8 x^{2}}} \Longrightarrow \max \{2 y, 2 z\}>t+\sqrt{t^{2}-8 x^{2}} .
$$


Since $t=y+z+a+b+c$ and $y z=2 x^{2}+x(a+b+c)+a c$ it is clear that (36) is symmetric in $y$ and $z$; therefore, without loss of generality we can assume that $\max \{2 y, 2 z\}=2 z$.

Consider now that $x$ and $z$ are fixed and $a, b, c$ variable. Then

$$
t=a+b+c+z+\frac{2 x^{2}+x(a+b+c)+a c}{z} \geq z+\frac{2 x^{2}}{z} .
$$

Since $t+\sqrt{t^{2}-8 x^{2}}$ is increasing in $t,(36)$ and (37) imply

$$
2 z>z+\frac{2 x^{2}}{z}+\sqrt{\left(z+\frac{2 x^{2}}{z}\right)^{2}-8 x^{2}}=z+\frac{2 x^{2}}{z}+\left|z-\frac{2 x^{2}}{z}\right| .
$$

The last inequality is obviously false. Therefore, our assumption that (35) does not hold leads to a contradiction. This ends Step 3 and with it the proof of Theorem 1.

\section{Proof of Theorem 2}

By (1) and (2), it is clear that (8) can be written in the equivalent form

$$
\frac{1}{A\left(H_{\min }\right)}+\frac{1}{A\left(h_{\max }\right)} \geq \frac{2}{A(K)}
$$

The main idea is to prove that

$$
\frac{1}{A(H)}+\frac{1}{A\left(h_{\max }\right)} \geq \frac{2}{A(D)}
$$

or equivalently that

$$
A(H) \leq \frac{A(D) \cdot A\left(h_{\max }\right)}{2 A\left(h_{\max }\right)-A(D)},
$$

where $H$ is some centrally symmetric hexagon circumscribed about $K$ and $D$ is some polygon inscribed in $K$. Clearly, since $A(D) \leq A(K)$ and $A(H) \geq A\left(H_{\min }\right)$, (39) would immediately imply (38). The following construction provides such candidates for $H$ and $D$.

Let $P_{1} P_{3} P_{5}\left(-P_{1}\right)\left(-P_{3}\right)\left(-P_{5}\right)=h_{\max }$ be a hexagon of maximum area inscribed in $K$. We then consider the support lines of $K$ which are parallel to the edges of $h_{\max }$; they determine a hexagon $H=Q_{1} Q_{3} Q_{5}\left(-Q_{1}\right)\left(-Q_{3}\right)\left(-Q_{5}\right)$ circumscribed about $K$. Denote the contact points between $H$ and $K$ by $P_{2}, P_{4}, P_{6},\left(-P_{2}\right),\left(-P_{4}\right),\left(-P_{6}\right)$ (see Fig. 2).

We denote by $D$ the dodecagon determined by the points $P_{i}$ and $-P_{i}, 1 \leq i \leq 6$. We will show that (39) holds true for this choice of $H$ and $D$.

As in the octagon case denote (see Fig. 3)

$$
\overline{P_{i} \bar{P}_{i+1}}=\mathrm{r}_{i}, \quad 1 \leq i \leq 6 \quad \text { (where } P_{7}=-P_{1} \text { ) }
$$




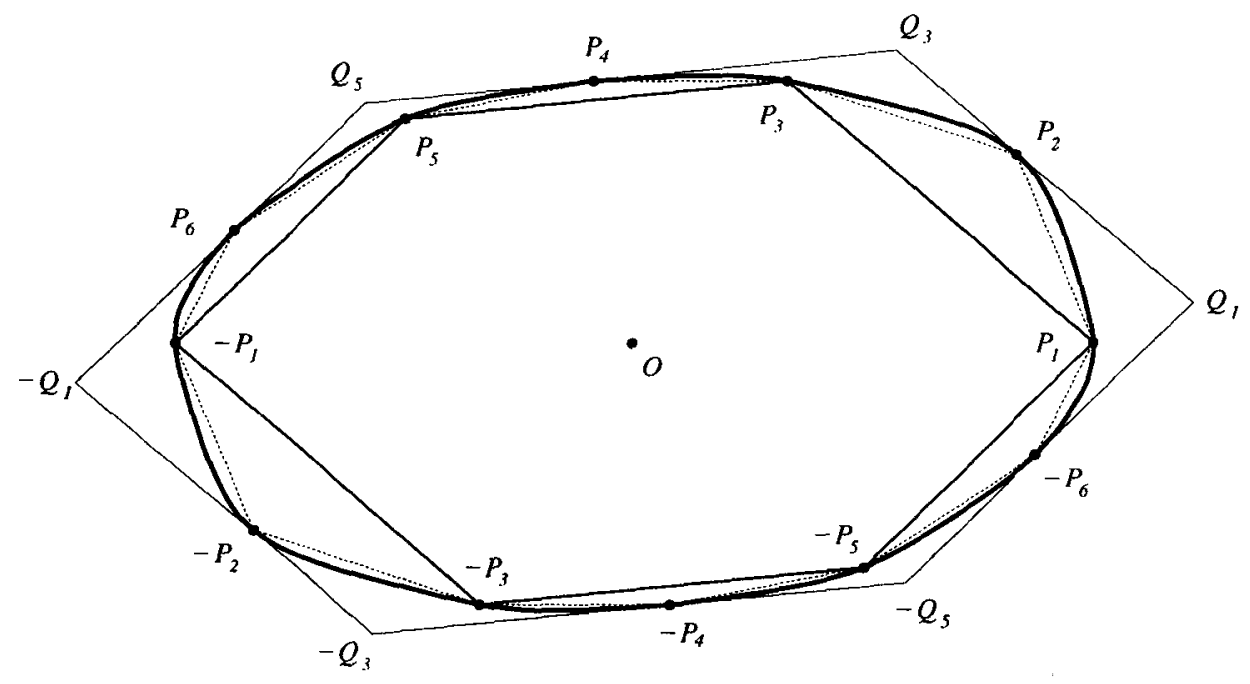

Fig. 2. The construction for Theorem 2.

and

$$
a_{i j}=\left(\mathbf{r}_{i}, \mathbf{r}_{j}\right), \quad 1 \leq i<j \leq 6 .
$$

A similar type of dependence between the $a_{i j}$ 's as in (13) can be proved; more precisely, for every $1 \leq i<j<k<l \leq 6$ we have

$$
a_{i j} a_{k l}-a_{i k} a_{j l}+a_{i l} a_{j k}=0
$$

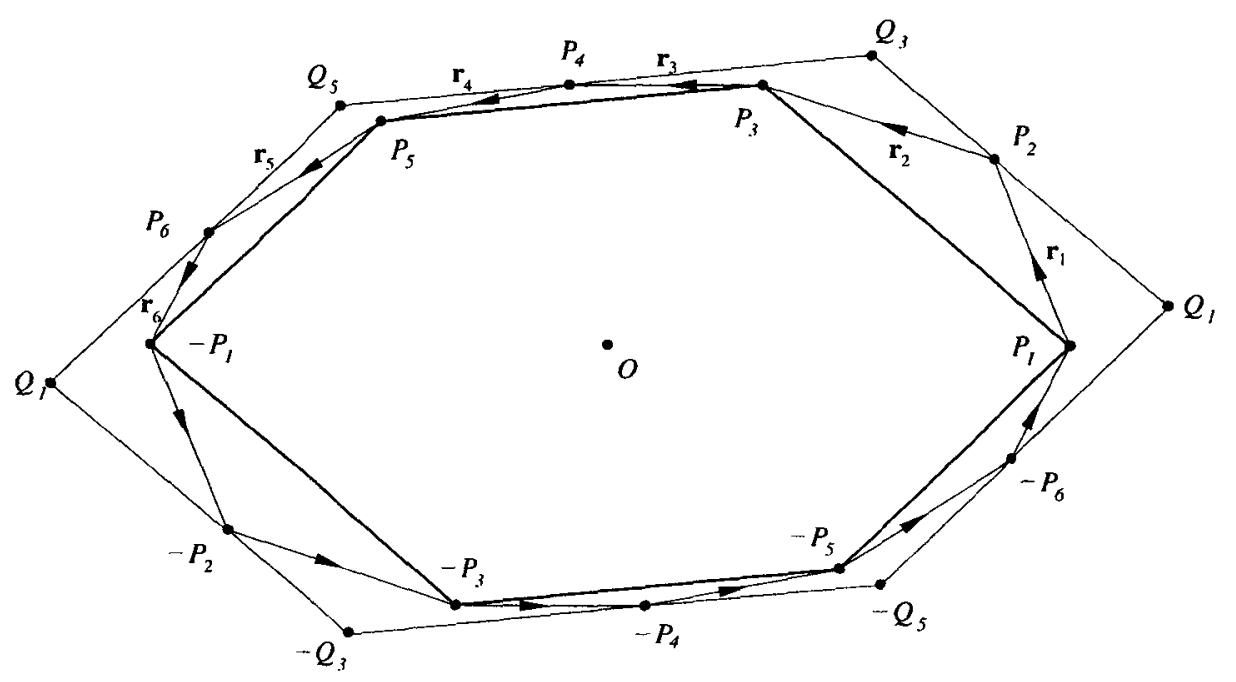

Fig. 3. The vectors $\mathbf{r}_{1}, \mathbf{r}_{2}, \ldots, \mathbf{r}_{6}$ determine the centrally symmetric dodecagon $\Delta$. 


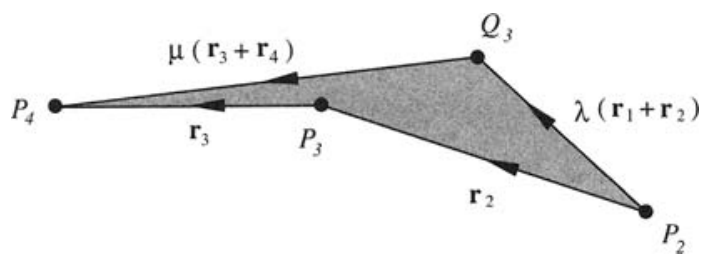

Fig. 4. An arrow-shaped region.

It easily follows that

$$
\begin{aligned}
A(D) & =\sum_{1 \leq i<j \leq 6} a_{i j}=S, \\
A\left(h_{\max }\right) & =S-\left(a_{12}+a_{34}+a_{56}\right) .
\end{aligned}
$$

Our next step would be to find an expression for the area of $H$; it is clear that $A(H)$ exceeds $A(D)$ by the area of six "arrow"-shaped regions: $P_{2} Q_{3} P_{4} P_{3}, P_{4} Q_{5} P_{6} P_{5}$, $\left(-P_{6}\right) Q_{1} P_{2} P_{1}$ and the remaining three are congruent with one of these.

We do the computations for the area of $\left(P_{2} Q_{3} P_{4} P_{3}\right)$ (see Fig. 4).

Since by construction $P_{2} Q_{3} \| P_{1} P_{3}$ and $Q_{3} P_{4} \| P_{3} P_{5}$ there exist some positive constants $\lambda$ and $\mu$ such that

$$
\overline{P_{2} Q_{3}}=\lambda\left(\mathbf{r}_{1}+\mathbf{r}_{2}\right) \text { and } \overline{Q_{3} P_{4}}=\mu\left(\mathbf{r}_{3}+\mathbf{r}_{4}\right)
$$

Now,

$$
\overline{P_{2} P_{4}}=\overline{P_{2} Q_{3}}+\overline{Q_{3} P_{4}}
$$

and the above relations give

$$
\mathbf{r}_{2}+\mathbf{r}_{3}=\lambda\left(\mathbf{r}_{1}+\mathbf{r}_{2}\right)+\mu\left(\mathbf{r}_{3}+\mathbf{r}_{4}\right)
$$

Multiplying this last relation first by $\mathbf{r}_{2}$ and then by $\mathbf{r}_{3}$ we obtain the following system:

$$
\begin{aligned}
& \lambda a_{12}-\mu\left(a_{23}+a_{24}\right)=-a_{23}, \\
& \lambda\left(a_{13}+a_{23}\right)-\mu a_{34}=a_{23} .
\end{aligned}
$$

Solving the above system for $\lambda$ we find (after using at some point (41) in the particular form $a_{12} a_{34}-a_{13} a_{24}+a_{14} a_{23}=0$ )

$$
\lambda=\frac{a_{23}+a_{24}+a_{34}}{a_{13}+a_{14}+a_{23}+a_{24}} .
$$

It follows that

$$
2 \cdot A\left(P_{2} Q_{3} P_{4}\right)=\left(\lambda\left(\mathbf{r}_{1}+\mathbf{r}_{2}\right), \mathbf{r}_{2}+\mathbf{r}_{3}\right)=\frac{\left(a_{12}+a_{13}+a_{23}\right)\left(a_{23}+a_{24}+a_{34}\right)}{a_{13}+a_{14}+a_{23}+a_{24}} .
$$

On the other hand,

$$
2 \cdot A\left(P_{2} P_{3} P_{4}\right)=a_{23}
$$


Then

$$
\begin{aligned}
2 \cdot A\left(P_{2} Q_{3} P_{4} P_{3}\right) & =2 \cdot A\left(P_{2} Q_{3} P_{4}\right)-2 \cdot A\left(P_{2} P_{3} P_{4}\right) \\
& =\frac{a_{12}\left(a_{23}+a_{24}+a_{34}\right)+a_{34}\left(a_{13}+a_{23}\right)+\left(a_{13} a_{24}-a_{14} a_{23}\right)}{a_{13}+a_{14}+a_{23}+a_{24}}
\end{aligned}
$$

from which we finally obtain

$$
2 \cdot A\left(P_{2} Q_{3} P_{4} P_{3}\right)=\frac{a_{12}\left(a_{23}+a_{24}+a_{34}\right)+a_{34}\left(a_{12}+a_{13}+a_{23}\right)}{a_{13}+a_{14}+a_{23}+a_{24}} .
$$

Similar computations lead to analogous expressions for the area of the other two arrow-shaped regions:

$$
\begin{aligned}
2 \cdot A\left(P_{4} Q_{5} P_{6} P_{5}\right) & =\frac{a_{34}\left(a_{45}+a_{46}+a_{56}\right)+a_{56}\left(a_{34}+a_{35}+a_{45}\right)}{a_{35}+a_{36}+a_{45}+a_{46}}, \\
2 \cdot A\left(\left(-P_{6}\right) Q_{1} P_{2} P_{1}\right) & =\frac{a_{56}\left(a_{12}+a_{16}+a_{26}\right)+a_{12}\left(a_{15}+a_{16}+a_{56}\right)}{a_{15}+a_{16}+a_{25}+a_{26}} .
\end{aligned}
$$

Now we are able to express $A(H)$ in terms of the $a_{i j}$ 's. However, we first introduce some useful notations.

For all triplets $1 \leq i<j<k \leq 6$ denote

$$
T_{i j k}=a_{i j}+a_{i k}+a_{j k}
$$

Also denote the denominators that occur in (44) and (45) as follows:

$$
\begin{aligned}
& a=a_{35}+a_{36}+a_{45}+a_{46}, \\
& b=a_{15}+a_{16}+a_{25}+a_{26}, \\
& c=a_{13}+a_{14}+a_{23}+a_{24} .
\end{aligned}
$$

Notice that (42), (43), and (47) immediately imply that

$$
A\left(h_{\max }\right)=S-\left(a_{12}+a_{34}+a_{56}\right)=a+b+c .
$$

With these new notations,

$$
A(H)=S+\frac{a_{12} T_{234}+a_{34} T_{123}}{c}+\frac{a_{34} T_{456}+a_{56} T_{345}}{a}+\frac{a_{56} T_{126}+a_{12} T_{156}}{b} .
$$

Now using (43) and (49), the inequality we would like to prove, (40), can be written in the equivalent form:

$$
\begin{gathered}
S+\frac{a_{12} T_{234}+a_{34} T_{123}}{c}+\frac{a_{34} T_{456}+a_{56} T_{345}}{a}+\frac{a_{56} T_{126}+a_{12} T_{156}}{b} \\
\leq \frac{S \cdot\left[S-\left(a_{12}+a_{34}+a_{56}\right)\right]}{S-2\left(a_{12}+a_{34}+a_{56}\right)}
\end{gathered}
$$


which after using (42) and (47) becomes

$$
\begin{array}{r}
\frac{a_{12} T_{234}+a_{34} T_{123}}{c}+\frac{a_{34} T_{456}+a_{56} T_{345}}{a}+\frac{a_{56} T_{126}+a_{12} T_{156}}{b} \\
\leq \frac{\left(a+b+c+a_{12}+a_{34}+a_{56}\right)\left(a_{12}+a_{34}+a_{56}\right)}{(a+b+c)-\left(a_{12}+a_{34}+a_{56}\right)} .
\end{array}
$$

We will show that an even stronger inequality than (50) is valid. Obviously, the proof of Theorem 2 will be finished after proving the following:

Claim. With the notations introduced above, we have

$$
\begin{array}{r}
\frac{a_{12} T_{234}+a_{34} T_{123}}{c}+\frac{a_{34} T_{456}+a_{56} T_{345}}{a}+\frac{a_{56} T_{126}+a_{12} T_{156}}{b} \\
\leq \frac{\left(a+b+c+a_{12}+a_{34}+a_{56}\right)\left(a_{12}+a_{34}+a_{56}\right)}{(a+b+c)} .
\end{array}
$$

Proof of the Claim. Multiplying both sides of (51) by $a b c$ the inequality to be proved becomes

$$
\begin{gathered}
a b\left(a_{12} T_{234}+a_{34} T_{123}\right)+b c\left(a_{34} T_{456}+a_{56} T_{345}\right)+a c\left(a_{56} T_{126}+a_{12} T_{156}\right) \\
-a b c\left(a_{12}+a_{34}+a_{56}\right) \leq \frac{a b c\left(a_{12}+a_{34}+a_{56}\right)^{2}}{a+b+c}
\end{gathered}
$$

which after rearranging the terms gives

$$
\begin{aligned}
& a a_{12}\left(b T_{234}+c T_{156}-b c\right)+b a_{34}\left(a T_{123}+c T_{456}-a c\right) \\
& +c a_{56}\left(a T_{126}+b T_{345}-a b\right) \leq \frac{a b c\left(a_{12}+a_{34}+a_{56}\right)^{2}}{a+b+c}
\end{aligned}
$$

The following identities are easy consequences of (41), (46), and (47):

$$
\begin{aligned}
& b T_{234}+c T_{156}-b c=-a \cdot a_{12}+b \cdot a_{34}+c \cdot a_{56}, \\
& a T_{123}+c T_{456}-a c=a \cdot a_{12}-b \cdot a_{34}+c \cdot a_{56}, \\
& b T_{345}+a T_{126}-a b=a \cdot a_{12}+b \cdot a_{34}-c \cdot a_{56} .
\end{aligned}
$$

Now using the identities above, (52) becomes

$$
\begin{aligned}
-a^{2} \cdot a_{12}^{2} & -b^{2} \cdot a_{34}^{2}-c^{2} \cdot a_{56}^{2}+2 a b \cdot a_{12} a_{34}+2 a c \cdot a_{12} a_{56}+2 b c \cdot a_{34} a_{56} \\
\leq & \frac{a b c\left(a_{12}+a_{34}+a_{56}\right)^{2}}{a+b+c} .
\end{aligned}
$$

Eliminating the denominator, (54) can now be written in the equivalent form

$$
\begin{aligned}
& a(a+b)(a+c) \cdot a_{12}^{2}-b(a+b)(b+c) \cdot a_{34}^{2}+c(a+c)(b+c) \cdot a_{56}^{2} \\
& -2 a b(a+b) \cdot a_{12} a_{34}-2 a c(a+c) \cdot a_{12} a_{56}-2 b c(b+c) \cdot a_{34} a_{56} \geq 0 .
\end{aligned}
$$

Regard (55) as a quadratic form in the variables $a_{12}, a_{34}, a_{56}$. In order to finish the proof it would be sufficient to show that this quadratic form is positive semidefinite. 
The associated matrix of this quadratic form is

$$
\left(\begin{array}{ccc}
a(a+b)(a+c) & -a b(a+b) & -a c(a+c) \\
-a b(a+b) & b(a+b)(b+c) & -b c(b+c) \\
-a c(a+c) & -b c(b+c) & c(a+c)(b+c)
\end{array}\right)
$$

For proving positive semidefiniteness it would be sufficient to show that all principal submatrices have nonnegative determinant. Recall from (47) that $a, b, c$ are positive numbers, that is, all $1 \times 1$ principal matrices have positive determinant. By the symmetry, it is enough to compute the determinant of the $2 \times 2$ upper left principal matrix: this is $a b c(a+b+c)(a+b)^{2}>0$. Finally, the determinant of the whole matrix is exactly zero. This proves (55) and with it the claim.

\section{Proof of Theorem 3}

Let $K$ have the origin $O$ as center of symmetry. The inequality (9) is trivially true if $K$ is a tile. Assume now that $\delta_{\mathrm{L}}(K)<1<\vartheta_{\mathrm{L}}(K)$. Denote by

$$
H_{\min }:=Q_{1} Q_{2} Q_{3}\left(-Q_{1}\right)\left(-Q_{2}\right)\left(-Q_{3}\right)
$$

a hexagon of minimum area circumscribed about $K$.

Let $P_{i}$ (resp. $-P_{i}$ ) be the intersection points between $\overline{O Q_{i}}$ and the boundary of $K$ (respectively between $\overline{O\left(-Q_{i}\right)}$ and the boundary of $K$ ) and let the constants $x, y, z \in$ $[0,1)$ be such that

$$
\begin{array}{llll}
\overline{P_{1} Q_{1}}=x \mathbf{r}_{1} & \overline{O P_{1}}=(1-x) \mathbf{r}_{1} & \text { where } & \mathbf{r}_{1}=\overline{O Q_{1}}, \\
\overline{P_{2} Q_{2}}=y \mathbf{r}_{2} & \overline{O P_{2}}=(1-y) \mathbf{r}_{2} & \text { where } & \mathbf{r}_{2}=\overline{O Q_{2}}, \\
\overline{P_{3} Q_{3}}=z \mathbf{r}_{3} & \overline{O P_{3}}=(1-z) \mathbf{r}_{3} & \text { where } & \mathbf{r}_{3}=\overline{O Q_{3}}
\end{array}
$$

We denote $h:=P_{1} P_{2} P_{3}\left(-P_{1}\right)\left(-P_{2}\right)\left(-P_{3}\right)$. Also, let $M_{i} N_{i}$ be the tangents at $K$ drawn at $P_{i}, i=1,2,3$. We thus obtain a dodecagon circumscribed about $K$ (see Fig. 5):

$$
D:=M_{1} N_{1} M_{2} N_{2} M_{3} N_{3}\left(-M_{1}\right)\left(-N_{1}\right)\left(-M_{2}\right)\left(-N_{2}\right)\left(-M_{3}\right)\left(-N_{3}\right) \text {. }
$$

It is useful to obtain an upper bound for $A(D)$. We first try to find a lower estimate for the area of $M_{2} Q_{2} N_{2}$. We have that

$$
\overline{P_{2} N_{2}}=y \mathbf{r}_{2}+\lambda\left(\mathbf{r}_{3}-\mathbf{r}_{2}\right) \text { and } \overline{M_{2} P_{2}}=-y \mathbf{r}_{2}+\mu\left(\mathbf{r}_{2}-\mathbf{r}_{1}\right) \quad \text { where } \quad \lambda, \mu \geq 0 \text {. }
$$

Since $M_{2}, P_{2}, N_{2}$ are collinear it follows that

$$
\begin{aligned}
\left(y \mathbf{r}_{2}+\lambda\left(\mathbf{r}_{3}-\mathbf{r}_{2}\right),-y \mathbf{r}_{2}+\mu\left(\mathbf{r}_{2}-\mathbf{r}_{1}\right)\right)=0 & \\
& \Longrightarrow \quad \mu y\left(\mathbf{r}_{2}, \mathbf{r}_{2}-\mathbf{r}_{1}\right)-\lambda y\left(\mathbf{r}_{3}-\mathbf{r}_{2}, \mathbf{r}_{2}\right)+\lambda \mu\left(\mathbf{r}_{3}-\mathbf{r}_{2}, \mathbf{r}_{2}-\mathbf{r}_{1}\right)=0 \\
& \Longrightarrow \quad \mu y a_{12}+\lambda y a_{23}-\lambda \mu\left(a_{12}-a_{13}+a_{23}\right)=0 \\
& \Longrightarrow \quad \mu=\frac{\lambda y a_{23}}{\lambda\left(a_{12}-a_{13}+a_{23}\right)-y a_{12}}
\end{aligned}
$$




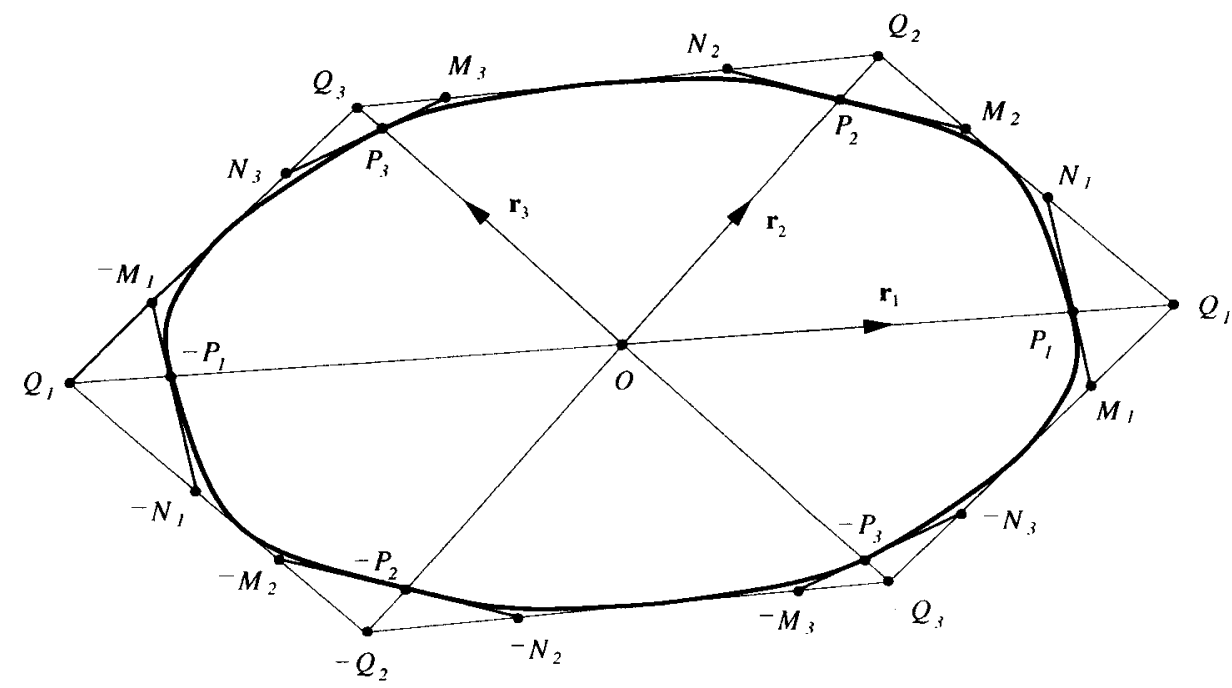

Fig. 5. The construction for Theorem 3 .

We have that

$$
2 A\left(M_{2} Q_{2} N_{2}\right)=\lambda \mu\left(\mathbf{r}_{2}-\mathbf{r}_{1}, \mathbf{r}_{3}-\mathbf{r}_{2}\right)=\frac{\lambda^{2} y a_{23}\left(a_{12}-a_{13}+a_{23}\right)}{\lambda\left(a_{12}-a_{13}+a_{23}\right)-y a_{12}} .
$$

A simple analysis shows that the minimum is attained for

$$
\lambda=\frac{2 y a_{12}}{a_{12}-a_{13}+a_{23}} .
$$

From this, an immediate calculation gives

$$
2 A\left(M_{2} Q_{2} N_{2}\right) \geq \frac{4 y^{2} a_{12} a_{23}}{a_{12}-a_{13}+a_{23}} .
$$

Analogously, it can be shown that

$$
\begin{gathered}
2 A\left(M_{1} Q_{1} N_{1}\right) \geq \frac{4 x^{2} a_{12} a_{13}}{a_{12}+a_{13}-a_{23}}, \\
2 A\left(M_{3} Q_{3} N_{3}\right) \geq \frac{4 z^{2} a_{13} a_{23}}{-a_{12}+a_{13}+a_{23}} .
\end{gathered}
$$

We now introduce some new variables given by

$$
a_{12}+a_{13}-a_{23}=2 a, \quad a_{12}-a_{13}+a_{23}=2 b, \quad-a_{12}+a_{13}+a_{23}=2 c .
$$

Notice that $a, b, c$ are all positive. For instance $b=\left(a_{12}+a_{23}-a_{13}\right) / 2=A\left(O Q_{1} Q_{2}\right)+A\left(O Q_{2} Q_{3}\right)-A\left(O Q_{1} Q_{3}\right)=A\left(Q_{1} Q_{2} Q_{3}\right)$. 
In these new variables the inequalities (57)-(59) become

$$
\begin{aligned}
& 2 A\left(M_{1} Q_{1} N_{1}\right) \geq \frac{2 x^{2}(a+b)(a+c)}{a}, \\
& 2 A\left(M_{2} Q_{2} N_{2}\right) \geq \frac{2 y^{2}(a+b)(b+c)}{b} \\
& 2 A\left(M_{3} Q_{3} N_{3}\right) \geq \frac{2 z^{2}(a+c)(b+c)}{c}
\end{aligned}
$$

We have that

$$
\begin{aligned}
A\left(H_{\min }\right) & =2\left[A\left(O Q_{1} Q_{2}\right)+A\left(O Q_{2} Q_{3}\right)+A\left(O Q_{3}\left(-Q_{1}\right)\right)\right] \\
& =a_{12}+a_{13}+a_{23}=2(a+b+c)
\end{aligned}
$$

hence,

$$
A\left(H_{\min }\right)=2(a+b+c)
$$

On the other hand since

$$
A\left(H_{\min }\right)-A(D)=2\left[A\left(M_{1} Q_{1} N_{1}\right)+A\left(M_{2} Q_{2} N_{2}\right)+A\left(M_{3} Q_{3} N_{3}\right)\right]
$$

by using (60) we obtain that

$$
\begin{aligned}
A\left(H_{\min }\right)-A(D) \geq & \frac{2 x^{2}(a+b)(a+c)}{a}+\frac{2 y^{2}(a+b)(b+c)}{b} \\
& +\frac{2 z^{2}(a+c)(b+c)}{c} .
\end{aligned}
$$

Now express the area of the hexagon $h:=P_{1} P_{2} P_{3}\left(-P_{1}\right)\left(-P_{2}\right)\left(-P_{3}\right)$ :

$$
\begin{aligned}
A(h) & =2\left[A\left(O P_{1} P_{2}\right)+A\left(O P_{2} P_{3}\right)+A\left(O P_{3}\left(-P_{1}\right)\right)\right] \\
& =(1-x)(1-y) a_{12}+(1-y)(1-z) a_{23}+(1-z)(1-x) a_{13}
\end{aligned}
$$

that is,

$$
A(h)=(1-x)(1-y)(a+b)+(1-x)(1-z)(a+c)+(1-y)(1-z)(b+c) .
$$

Now using (61) and the expression of $A(h)$ we obtain

$$
\begin{aligned}
A\left(H_{\min }\right)-A(h)= & a(2 x+y+z-x y-x z)+b(x+2 y+z-x y-y z) \\
& +c(x+y+2 z-x z-y z) .
\end{aligned}
$$

From this it follows at once that

$$
\begin{aligned}
A\left(H_{\min }\right)-A(h) & \leq a(2 x+y+z)+b(x+2 y+z)+c(x+y+2 z) \\
& =x(2 a+b+c)+y(a+2 b+c)+z(a+b+2 c) .
\end{aligned}
$$


Claim B. For all positive $a, b, c$ and all real numbers $x, y, z$ the following inequality holds:

$$
\begin{gathered}
5(a+b+c)\left[x^{2} \frac{(a+b)(a+c)}{a}+y^{2} \frac{(a+b)(b+c)}{b}+z^{2} \frac{(a+c)(b+c)}{c}\right] \\
-[x(2 a+b+c)+y(a+2 b+c)+z(a+b+2 c)]^{2} \geq 0 .
\end{gathered}
$$

A proof of this claim is given in the Appendix.

Now using (61)-(63) and Claim B it follows easily that

$$
\frac{5}{4} A\left(H_{\min }\right)\left[A\left(H_{\min }-A(D)\right] \geq\left[A\left(H_{\min }\right)-A(h)\right]^{2} .\right.
$$

Dividing both sides by $A(K)^{2}$ we obtain

$$
\frac{5}{4} \frac{A\left(H_{\min }\right)}{A(K)}\left[\frac{A\left(H_{\min }\right)}{A(K)}-\frac{A(D)}{A(K)}\right] \geq\left[\frac{A\left(H_{\min }\right)}{A(K)}-\frac{A(h)}{A(K)}\right]^{2}
$$

However, we know that

$$
\frac{1}{\delta_{\mathrm{L}}(K)}=\frac{A\left(H_{\min }\right)}{A(K)}, \quad \frac{1}{\vartheta_{\mathrm{L}}(K)} \leq \frac{A(h)}{A(K)}, \quad 1 \leq \frac{A(D)}{A(K)} .
$$

Using this relation in the previous inequality we obtain that

$$
\begin{aligned}
\frac{5}{4} \frac{1}{\delta_{\mathrm{L}}(K)}\left[\frac{1}{\delta_{\mathrm{L}}(K)}-1\right] \geq\left[\frac{1}{\delta_{\mathrm{L}}(K)}-\frac{1}{\vartheta_{\mathrm{L}}(K)}\right]^{2} \\
\quad \Longrightarrow \quad \vartheta_{\mathrm{L}}(K)^{2}\left(5 \delta_{\mathrm{L}}(K)-1\right)-8 \delta_{\mathrm{L}}(K) \vartheta_{\mathrm{L}}(K)+4 \delta_{\mathrm{L}}(K)^{2} \leq 0 \\
\quad \Longrightarrow \quad \vartheta_{\mathrm{L}}(K) \leq \frac{4 \delta_{\mathrm{L}}(K)+2 \sqrt{5} \delta_{\mathrm{L}}(K) \sqrt{1-\delta_{\mathrm{L}}(K)}}{5 \delta_{\mathrm{L}}(K)-1} \\
\Longrightarrow \quad \frac{\vartheta_{\mathrm{L}}(K)-1}{\sqrt{1-\delta_{\mathrm{L}}(K)}} \leq \frac{\sqrt{1-\delta_{\mathrm{L}}(K)}+2 \sqrt{5} \delta_{\mathrm{L}}(K)}{5 \delta_{\mathrm{L}}(K)-1}
\end{aligned}
$$

By (3), $\delta_{\mathrm{L}}(K) \geq 0.89$ for every centrally symmetric plane convex body $K$. The function $f:[0.89,1] \rightarrow \mathbf{R}, f(t)=(\sqrt{1-t}+2 \sqrt{5} t) /(5 t-1)$ is decreasing, hence

$$
\frac{\sqrt{1-\delta_{\mathrm{L}}(K)}+2 \sqrt{5} \delta_{\mathrm{L}}(K)}{5 \delta_{\mathrm{L}}(K)-1} \leq f(0.89)<1.25
$$

Therefore

$$
\vartheta_{\mathrm{L}}(K)-1 \leq 1.25 \sqrt{1-\delta_{\mathrm{L}}(K)}
$$

which is the desired inequality. 


\section{Proofs of Theorems 4 and 5}

We use the description of $\Omega_{\mathrm{L}}\left(\mathcal{P}_{8}\right)$ proved in Theorem 1. If in (7) we expand in Taylor series near the point $(\vartheta, \delta)=(1,1)$ we obtain that for every $K \in \mathcal{P}_{8}$ we have

$$
\begin{aligned}
& (\vartheta-1)-2(\vartheta-1)^{2}-4(\vartheta-1)^{3}+O\left((\vartheta-1)^{3}\right) \\
& \geq 1-\delta \geq(\vartheta-1)^{2}+2(\vartheta-1)^{3}+O\left((\vartheta-1)^{3}\right),
\end{aligned}
$$

where $\delta$ and $\vartheta$ stand for $\delta_{\mathrm{L}}(K)$ and $\vartheta_{\mathrm{L}}(K)$, respectively.

Moreover, since in (7) we have equality, the leading coefficients in (64) cannot be replaced by stronger ones. Therefore, statements (10) and (11) are true for appropriately chosen $K_{\varepsilon}$ 's in $\mathcal{P}_{8}$.

\section{Conclusions and Open Problems}

The density bounds mentioned in the Introduction and the results proved in the previous sections can be summarized by a graphic illustration (see Fig. 6).

The problem of giving a complete and explicit description of the set $\Omega_{\mathrm{L}}(\mathcal{C})$, where $\mathcal{C}$ is the class of all centrally symmetric convex bodies, seems to be very difficult. Here is a list of presumably simpler questions (some of which were first raised in [10]) which may have a better chance of being answered first:

Question 1. Is it possible to replace the " 1.25 " coefficient appearing in (9) by "1"?

Question 2. Determine $\Omega_{\mathrm{L}}\left(\mathcal{P}_{10}\right)$. Notice that the "apex" of the set describing $\Omega_{\mathrm{L}}\left(\mathcal{P}_{8}\right)$, the point $O_{\text {reg }}=\left(\frac{4}{7}(3-\sqrt{2}), 4-2 \sqrt{2}\right)$, corresponds to the (affinely) regular octagon.

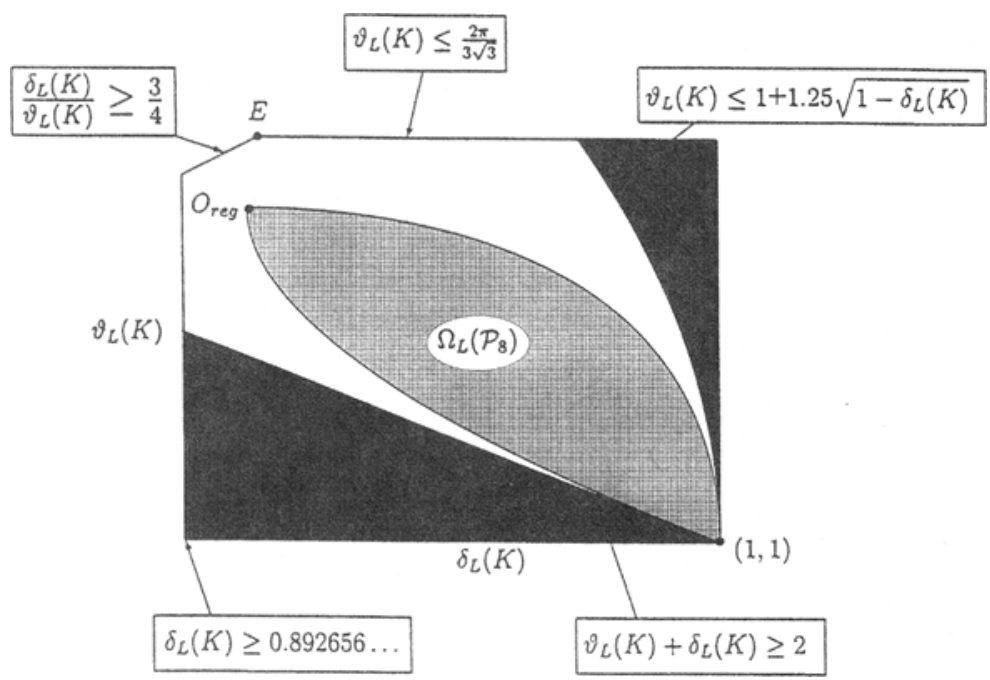

Fig. 6. Region enclosing $\Omega_{\mathrm{L}}(\mathcal{C})$. 
However, note that in [11] it is proved that the centrally symmetric decagon with minimal packing density is not the regular one.

Question 3. For which values of $n \operatorname{are} \min \delta_{\mathrm{L}}(K)$ and $\max \vartheta_{\mathrm{L}}(K)$ achieved for the same $K$ ? (Here the minimum and the maximum are taken over all $K \in \mathcal{P}_{2 n}$.) More generally, what is the "geometry" of $\Omega_{\mathrm{L}}\left(\mathcal{P}_{2 n}\right)$ for $\delta$ "small" and $\vartheta$ "large"?

Question 4. Is the set $\Omega_{\mathrm{L}}(\mathcal{C})$ simply connected? Is it convex?

Question 5. Is the point $E=(\pi / \sqrt{12}, 2 \pi / \sqrt{27})$ corresponding to ellipses, the only point of $\Omega_{\mathrm{L}}(\mathcal{C})$ which lies on the line $\delta=\frac{3}{4} \vartheta$ ?

Question 6. Is the "smoothed-octagon" first constructed by Reinhardt the centrally symmetric convex body of minimal packing density? An improvement of (3) would also be desirable.

\section{Appendix}

Proof of Claim A. Using (18) and (19), the first equality (i.e., (21)) becomes equivalent to $\alpha \geq 1$. We consider the quadratic function whose greatest zero is $\alpha$ :

$$
f(x)=x^{2} \cdot(\vartheta-\delta)^{2}-x \cdot \delta \vartheta(1-\delta)(2-\vartheta)+\vartheta^{2}(1-\delta)^{2} .
$$

In order to prove that $\alpha \geq 1$ it would be sufficient to show that $f(1) \leq 0$, which after some computation gives

$$
\delta^{2}(2 \vartheta+1)-\delta\left(4 \vartheta+\vartheta^{2}\right)+2 \vartheta^{2} \leq 0
$$

that is,

$$
\frac{\vartheta\left(\vartheta+4-\sqrt{\vartheta^{2}-8 \vartheta+8}\right)}{4 \vartheta+2} \leq \delta \leq \frac{\vartheta\left(\vartheta+4+\sqrt{\vartheta^{2}-8 \vartheta+8}\right)}{4 \vartheta+2} .
$$

The right inequality is true by the definition of $U$; for the left one it would suffice to show that

$$
\frac{\vartheta\left(\vartheta+4-\sqrt{\vartheta^{2}}-8 \vartheta+8\right)}{4 \vartheta+2} \leq \frac{5 \vartheta^{2}-12 \vartheta+8}{2 \vartheta^{2}-5 \vartheta+4},
$$

which after some calculations can be written in the following form:

$$
\vartheta\left(2 \vartheta^{2}-5 \vartheta+4\right) \sqrt{\vartheta^{2}-8 \vartheta+8}+\left(\vartheta^{2}-8 \vartheta+8\right)\left(2+\vartheta-2 \vartheta^{2}\right) \geq 0 .
$$

Since $\vartheta \in[1,4-2 \sqrt{2}]$ it can be easily seen that both terms of the left side of the last relation are nonnegative. This proves $(21)$.

For proving the second part of the claim, notice first by using (18) that (22) is equivalent to

$$
\frac{\alpha^{2}}{\alpha^{2}+1} \geq \frac{\vartheta^{2}(1-\delta)^{2}}{\delta^{2}(\vartheta-1)^{2}}
$$


Consider the quadratic equation (19):

$$
x^{2} \cdot(\vartheta-\delta)^{2}-x \cdot \delta \vartheta(1-\delta)(2-\vartheta)+\vartheta^{2}(1-\delta)^{2}=0
$$

with roots $\alpha, \beta$ where $\alpha \geq \beta$. It is an elementary exercise to construct the quadratic equation with roots $\alpha^{2} /\left(\alpha^{2}+1\right)$ and $\beta^{2} /\left(\beta^{2}+1\right)$ :

$$
\begin{aligned}
g(x)= & x^{2} \cdot \delta^{2}\left[(\vartheta-1)^{2}(2 \vartheta-\vartheta \delta-\delta)^{2}+\vartheta^{2}(1-\delta)^{2}(2-\vartheta)^{2}\right] \\
& -x \cdot \vartheta^{2} \delta(1-\delta)^{2}\left[\delta\left(3 \vartheta^{2}-4 \vartheta+2\right)-4 \vartheta(\vartheta-1)\right]+\vartheta^{4}(1-\delta)^{4}=0 .
\end{aligned}
$$

For proving (65) it is sufficient to show that

$$
g\left(\frac{\vartheta^{2}(1-\delta)^{2}}{\delta^{2}(\vartheta-1)^{2}}\right) \leq 0
$$

which after some extensive but straightforward calculations becomes

$$
\delta^{2}\left(2 \vartheta^{2}-5 \vartheta+4\right)-2 \delta\left(\vartheta^{3}-4 \vartheta+4\right)+\vartheta\left(5 \vartheta^{2}-12 \vartheta+8\right) \leq 0
$$

that is,

$$
\frac{5 \vartheta^{2}-12 \vartheta+8}{2 \vartheta^{2}-5 \vartheta+4} \leq \delta \leq \vartheta
$$

The left inequality is satisfied by our definition of $U$; the right one is just obvious.

Proof of Claim $B$. For all positive $a, b, c$ we need to show that

$$
\begin{aligned}
5(a+b+c) & {\left[x^{2} \frac{(a+b)(a+c)}{a}+y^{2} \frac{(a+b)(b+c)}{b}+z^{2} \frac{(a+c)(b+c)}{c}\right] } \\
- & {[x(2 a+b+c)+y(a+2 b+c)+z(a+b+2 c)]^{2} \geq 0 . }
\end{aligned}
$$

Regard the left-hand side of the last relation as a quadratic form in the variables $x, y, z$. We want to show that this is positive definite.

We introduce some shorthand notations:

$$
S=a+b+c ; \quad T=a b+a c+b c ; \quad P=a b c .
$$

The following relations will be useful later:

$$
\begin{aligned}
& a^{2}+b^{2}+c^{2}=S^{2}-2 T \\
& a^{3}+b^{3}+c^{3}=S^{3}-3 S T+3 P \\
& a^{4}+b^{4}+c^{4}=S^{4}-4 S^{2} T+2 T^{2}+4 S P
\end{aligned}
$$


The associated matrix of this quadratic form is

$$
\left(\begin{array}{ccc}
\frac{5 S(a+b)(a+c)}{a}-(S+a)^{2} & -(S+a)(S+b) & -(S+a)(S+c) \\
-(S+a)(S+b) & \frac{5 S(a+b)(b+c)}{b}-(S+b)^{2} & -(S+b)(S+c) \\
-(S+a)(S+c) & -(S+b)(S+c) & \frac{5 S(a+c)(b+c)}{c}-(S+c)^{2}
\end{array}\right) \text {. }
$$

A necessary and sufficient condition for the positive definiteness of a matrix is that the minors $\Delta_{i}$, obtained by taking the first $i$ rows and the first $i$ columns, are all positive $(i=1,2,3)$ :

$$
\begin{aligned}
& \Delta_{1}=\frac{5 S(a+b)(a+c)}{a}-(S+a)^{2} \\
& \Longrightarrow \quad \Delta_{1}=5 S\left(S+\frac{b c}{a}\right)-(S+a)^{2}>5 S^{2}-(2 S)^{2}=S^{2}>0 .
\end{aligned}
$$

On the other hand,

$$
\begin{aligned}
\Delta_{2} & =\left|\begin{array}{cc}
\frac{5 S(a+b)(a+c)}{a}-(S+a)^{2} & -(S+a)(S+b) \\
-(S+a)(S+b) & \frac{5 S(a+b)(b+c)}{b}-(S+b)^{2}
\end{array}\right| \\
& =\frac{5 S(a+b)}{a b} \cdot\left[5 S(a+b)(a+c)(b+c)-b(a+c)(S+b)^{2}-a(b+c)(S+a)^{2}\right] .
\end{aligned}
$$

It follows that

$$
\begin{aligned}
\frac{a b \Delta_{2}}{5 S(a+b)}= & 5 S(S-a)(S-b)(S-c)-b(S-b)(S+b)^{2}-a(S-a)(S+a)^{2} \\
= & 5 S^{2}(a b+a c+b c)-5 S a b c-(a+b) S^{3} \\
& -\left(a^{2}+b^{2}\right) S^{2}+\left(a^{3}+b^{3}\right) S+\left(a^{4}+b^{4}\right) .
\end{aligned}
$$

Now using relations (66) it follows after some work that

$$
\frac{a b \Delta_{2}}{5 S(a+b)}=2 S P+2 T^{2}+c(S-c)(S+c)^{2}>0 \text {. }
$$

Hence, $\Delta_{2}>0$.

Finally, the same type of computations will give that

$$
\begin{aligned}
\frac{a b c \Delta_{3}}{25 S^{2}(a+b)(a+c)(b+c)}= & 5 S(S T-P)-(a+b+c) S^{3} \\
& -\left(a^{2}+b^{2}+c^{2}\right) S^{2}+\left(a^{3}+b^{3}+c^{3}\right) S+\left(a^{4}+b^{4}+c^{4}\right)
\end{aligned}
$$

and after using the relations (66) we obtain

$$
\frac{a b c \Delta_{3}}{25 S^{2}(a+b)(a+c)(b+c)}=2 S P+2 T^{2}>0 .
$$

Hence, $\Delta_{3}>0$.

This proves that all three minors $\Delta_{1}, \Delta_{2}, \Delta_{3}$ are positive and ends the proof of the claim. 


\section{References}

1. C. H. Dowker, On minimum circumscribed polygons, Bull. Amer. Math. Soc. 50 (1944), 120-122.

2. V. Ennola, On the lattice constant of a symmetric convex domain, J. London Math. Soc. 36 (1961), 135-138.

3. G. Fejes Tóth and W. Kuperberg, Packing and covering with convex sets, in Handbook of Convex Geometry, edited by P. M. Gruber and J. M. Wills, North-Holland, Amsterdam, 1993, pp. 799-860.

4. G. Fejes Tóth and W. Kuperberg, A survey of recent results in the theory of packing and covering, in New Trends in Discrete and Computational Geometry, Algorithms Combin., 10, Springer-Verlag, Berlin, 1993, pp. 251-279.

5. L. Fejes-Tóth, Some packing and covering theorems, Acta Sci. Math. (Szeged) 12/A (1950), 62-67.

6. L. Fejes-Tóth, Regular Figures, Pergamon Press, New York, 1964.

7. L. Fejes-Tóth, Lagerungen in der Ebene, auf Kugel und im Raum, Springer-Verlag, Berlin, 1972.

8. H. Groemer, Existenzsätze für Lagerungen im Euklidischen Raum, Math. Z. 81 (1963), 260-278.

9. M. Hausner, A Vector Space Approach to Geometry, Prentice-Hall, Englewood Cliffs, NJ, 1965.

10. W. Kuperberg, An inequality linking packing and covering densities of plane convex bodies, Geom. Dedicata 23 (1987), 59-66.

11. W. Ledermann and K. Mahler, On lattice points in a convex decagon, Acta Math. 81 (1949), 319-351.

12. K. Mahler, On the minimum determinant and the circumscribed hexagons of a convex domain, Nederl. Akad. Wetensch. Proc. 50 (1947), 692-703.

13. J. Pach and P. K. Agarwal, Combinatorial Geometry, Series in Discrete Mathematics and Optimization, Wiley Interscience, New York, 1995.

14. K. Reinhardt, Über die dichteste gitterförmige Lagerung kongruente Bereiche in der Ebene und eine besondere Art konvexer Kurven, Abh. Mathematischen Seminar, Hamburg, Hansischer Universität 10 (1934), 216-230.

15. E. Sas, Über eine Extremumeigenschaft der Ellipsen, Compositio Math. 6 (1939), 468-470.

16. W. M. Schmidt, Zur Lagerung kongruenter Körper im Raum, Monatsh. Math. 65 (1961), 154-158.

17. P. Tammela, An estimate of the critical determinant of a two-dimensional convex symmetric domain (Russian), Izv. Vyss. Uchebn. Zaved. Mat. 12 (1970), 103-107.

Received November 30, 1999, and in revised form April 24, 2000. Online publication September 6, 2000. 